\title{
Time-dependent exchange-correlation functional for a Hubbard dimer: Quantifying nonadiabatic effects
}

\author{
Johanna I. Fuks, ${ }^{1,2,}{ }^{*}$ Mehdi Farzanehpour, ${ }^{1, *}$ Ilya V. Tokatly, ${ }^{1,3}$ Heiko Appel, ${ }^{4}$ Stefan Kurth,,${ }^{1,3}$ and Angel Rubio ${ }^{1,4}$ \\ ${ }^{1}$ Nano-Bio Spectroscopy Group and ETSF, Departamento Física de Materiales, Universidad del País Vasco, Centro de Física de Materiales \\ CSIC-UPVIEHU-MPC and DIPC, Av. Tolosa 72, E-20018 San Sebastián, Spain \\ ${ }^{2}$ Department of Physics and Astronomy, Hunter College and the Graduate Center of the City University of New York, New York 10065, USA \\ ${ }^{3}$ IKERBASQUE, Basque Foundation for Science, E-48011 Bilbao, Spain \\ ${ }^{4}$ Fritz-Haber-Institut der Max-Planck-Gesellschaft, Faradayweg 4-6, D-14195 Berlin, Germany
}

(Received 22 November 2013; published 23 December 2013)

\begin{abstract}
We address and quantify the role of nonadiabaticity ("memory effects") in the exchange-correlation (xc) functional of time-dependent density functional theory (TDDFT) for describing nonlinear dynamics of many-body systems. Time-dependent resonant processes are particularly challenging for available TDDFT approximations, due to their strong nonlinear and nonadiabatic character. None of the known approximate density functionals are able to cope with this class of problems in a satisfactory manner. In this work we look at the prototypical example of the resonant processes by considering Rabi oscillations within the exactly soluble two-site Hubbard model. We construct the exact adiabatic xc functional and show that (i) it does not reproduce correctly resonant Rabi dynamics, and (ii) there is a sizable nonadiabatic contribution to the exact xc potential, which turns out to be small only at the beginning and at the end of the Rabi cycle when the ground-state population is dominant. We then propose a "two-level" approximation for the time-dependent xc potential which can capture Rabi dynamics in the two-site problem. It works well both for resonant and for detuned Rabi oscillations and becomes essentially exact in the linear response regime.
\end{abstract}

DOI: 10.1103/PhysRevA.88.062512

PACS number(s): 31.15.ee, 42.65.-k, 71.15.Mb

\section{INTRODUCTION}

Due to the favorable balance between efficiency and accuracy, time-dependent density functional theory (TDDFT) is becoming the theory of choice to describe the interaction of many-electron systems with external electromagnetic fields of arbitrary intensity, shape, and time dependence. Within this theory the observables are expressible as functionals of the time-dependent density. Similarly to static DFT, in TDDFT one can define an auxiliary noninteracting Kohn-Sham (KS) system which reproduces the exact time-dependent dynamics of the density. It is the propagation of this auxiliary system in an (unknown) effective local potential which makes TDDFT computationally powerful. However, despite the great success of the theory in describing optical properties of a large variety of molecules and nanostructures [1-3], the available approximations for the exchange-correlation (xc) potential exhibit serious deficiencies in the description of long range charge transfer [4-6], double excitations [7-9], and timeresolved nonlinear dynamics (see shortly), to mention a few.

The theoretical challenge is to improve the available functionals in order to capture the nonlocality both in space and time of the exact xc functional which depends on the entire history of the density, the initial (interacting) many-body state, and the initial KS state [10-14].

We note that almost all TDDFT calculations today use an adiabatic approximation for the xc potential, which uses the instantaneous density as input for an approximate groundstate functional. Thus, this approximation completely neglects

\footnotetext{
${ }^{*}$ These authors contributed equally to this work.
}

both the history and the initial-state dependence of the exact functional.

The successes and failures of the adiabatic approximation to describe linear response phenomena have been addressed in many works [1,2,15-17]. However, much less is known about the performance of adiabatic TDDFT for general dynamics beyond linear response. In some of our past studies $[8,11,18,19]$ on one-dimensional model systems we have shown that adiabatic xc functionals fail to describe dynamical processes where the density changes significantly in time (e.g., in photophysical and chemical processes where valence electrons are promoted to empty states). There are few cases where the exact time-dependent $\mathrm{xc}$ potential is known and can thus be used to test approximations [11,20,21]. In these works it has been shown numerically that novel dynamical steps appear in the xc potential which are fundamental to capture the proper resonant versus nonresonant dynamics and charge localization. While the construction of accurate approximations to the exact universal xc functional of TDDFT for Coulomb systems remains a challenge, simple model Hamiltonians constitute a convenient framework to gain insights into the properties of the exact TDDFT functional.

In the present work we exploit the possibilities of a solvable lattice model-the two-site Hubbard model [2224] - to address the impact of nonlocality in time in the exchange-correlation functional of TDDFT. Specifically, we study resonant Rabi oscillations, a prototypical example of nonlinear external field driven dynamics where the population of states changes dramatically in time. We first derive here the exact ground-state Hartree-exchange-correlation (Hxc) functional for the two-site model using the Levy-Lieb constrained search [25-27]. This functional, when used in a TDDFT context with the instantaneous time-dependent density as 
input, constitutes the exact adiabatic approximation which can be used as a reference to quantify the role of memory effects. By carefully studying and quantifying the dynamics produced by TDDFT with the adiabatic Hxc potential we demonstrate that it fails both quantitatively and qualitatively to describe Rabi oscillations. In the second part of this work we apply an analytic density-potential map for lattice systems [10,28] to derive an explicit, fully nonadiabatic xc density functional which correctly captures all features of Rabi dynamics in the Hubbard dimer. This functional is one of the main results of this paper.

The paper is organized as follows: In Sec. II we introduce the physics of the Rabi effect for the Hubbard dimer, showing how the dipole moment and state occupations evolve with time during the course of resonant Rabi oscillations. In Sec. III we address the same problem from a TDDFT perspective. In particular, we use the exact adiabatic xc functional as a reference to quantify memory effects. In Sec. IV we consider the exact interacting system in a two-level approximation which allows us to derive a new approximate Hxc potential as an explicit functional of the time-dependent density. The excellent performance of this approximation is demonstrated and explained. We end the paper with our conclusions in Sec. V. In the Appendix we derive the exact ground-state xc potential for the Hubbard dimer using the Levy-Lieb constrained search.

\section{RABI OSCILLATIONS FOR TWO-SITE HUBBARD MODEL}

We consider the dynamics of two electrons on a Hubbard dimer, that is, a two-site interacting Hubbard model with onsite repulsion $U$ and hopping parameter $T$. The Hamiltonian of the system reads

$$
\begin{aligned}
\hat{H}= & -T \sum_{\sigma}\left(\hat{c}_{1 \sigma}^{\dagger} \hat{c}_{2 \sigma}+\hat{c}_{2 \sigma}^{\dagger} \hat{c}_{1 \sigma}\right)+U\left(\hat{n}_{1 \uparrow} \hat{n}_{1 \downarrow}+\hat{n}_{2 \uparrow} \hat{n}_{2 \downarrow}\right) \\
& +\sum_{\sigma}\left[v_{1}(t) \hat{n}_{1 \sigma}+v_{2}(t) \hat{n}_{2 \sigma}\right]
\end{aligned}
$$

where $\hat{c}_{i \sigma}^{\dagger}$ and $\hat{c}_{i \sigma}$ are creation and annihilation operators for a spin- $\sigma$ electron on site $i$, respectively. The $\hat{n}_{i \sigma}=\hat{c}_{i \sigma}^{\dagger} \hat{c}_{i \sigma}$ are the operators for the spin- $\sigma$ density at site $i$, and the $v_{1,2}(t)$ are time-dependent on-site potentials. We use $\hbar=e=1$ throughout this work. Energies are given in units of the hopping parameter $T$. As we will see, this simple model captures most qualitative features of Rabi oscillations in interacting systems as well as the main difficulties of describing Rabi dynamics within TDDFT.

The many-body time-dependent Schrödinger equation

$$
i \partial_{t}|\psi(t)\rangle=H(t)|\psi(t)\rangle
$$

describes the evolution of the system from a given initial state $\left|\psi_{0}\right\rangle$. Since the Hamiltonian (1) is independent of spin, the spin structure of the wave function $|\psi(t)\rangle$ is fixed by the initial state. In the following we study the evolution from the ground state of the Hubbard dimer and therefore it is sufficient to consider only the singlet sector of our model.
In the absence of an external potential, $v_{1,2}=0 \mathrm{~T}$, the stationary singlet eigenstates of the Hamiltonian (1) take the form

$$
\begin{aligned}
& |g\rangle=\mathcal{N}_{g}\left[\hat{c}_{1 \uparrow}^{\dagger} \hat{c}_{1 \downarrow}^{\dagger}+\hat{c}_{2 \uparrow}^{\dagger} \hat{c}_{2 \downarrow}^{\dagger}+\beta_{+}\left(\hat{c}_{1 \uparrow}^{\dagger} \hat{c}_{2 \downarrow}^{\dagger}-\hat{c}_{1 \downarrow}^{\dagger} \hat{c}_{2 \uparrow}^{\dagger}\right)\right]|0\rangle, \\
& \left|e_{1}\right\rangle=1 / \sqrt{2}\left(\hat{c}_{1 \uparrow}^{\dagger} \hat{c}_{1 \downarrow}^{\dagger}+\hat{c}_{2 \uparrow}^{\dagger} \hat{c}_{2 \downarrow}^{\dagger}\right)|0\rangle, \\
& \left|e_{2}\right\rangle=\mathcal{N}_{e_{2}}\left[\hat{c}_{1 \uparrow}^{\dagger} \hat{c}_{1 \downarrow}^{\dagger}+\hat{c}_{2 \uparrow}^{\dagger} \hat{c}_{2 \downarrow}^{\dagger}+\beta_{-}\left(\hat{c}_{1 \uparrow}^{\dagger} \hat{c}_{2 \downarrow}^{\dagger}-\hat{c}_{1 \downarrow}^{\dagger} \hat{c}_{2 \uparrow}^{\dagger}\right)\right]|0\rangle .
\end{aligned}
$$

Here $|0\rangle$ is the vacuum state, $|g\rangle$ is the ground state, and $\left|e_{1,2}\right\rangle$ are two excited singlet states. The $\mathcal{N}_{g / e_{2}}=\left(2+2 \beta_{ \pm}^{2}\right)^{-1 / 2}$ are normalization factors, and the coefficients $\beta_{ \pm}$are defined as

$$
\beta_{ \pm}=\left(U \pm \sqrt{16 T^{2}+U^{2}}\right) / 4 T \text {. }
$$

The energy eigenvalues corresponding to the eigenstates (3) are

$$
\begin{aligned}
E_{g} & =2 T \beta_{-}, \\
E_{e_{1}} & =U, \\
E_{e_{2}} & =2 T \beta_{+} .
\end{aligned}
$$

To simplify notations, we rewrite the external potential part in Eq. (1) in the form

$$
\sum_{\sigma}\left(v_{1} \hat{n}_{1 \sigma}+v_{2} \hat{n}_{2 \sigma}\right)=\frac{\Delta v}{2}\left(\hat{n}_{1}-\hat{n}_{2}\right)+C(t)\left(\hat{n}_{1}+\hat{n}_{2}\right),
$$

where $\hat{n}_{i}=\sum_{\sigma} \hat{n}_{i \sigma}$ is the operator of the number of particles on site $i, \Delta v=v_{1}-v_{2}$ is the difference of on-site potentials, and $C(t)=\left[v_{1}(t)+v_{2}(t)\right] / 2$. The last term in Eq. (6) corresponds to a spatially uniform potential. This term can be trivially gauged away and will be ignored in the following without loss of generality. Nontrivial physical effects come only from the external potential $\Delta v$ which is coupled to the difference of on-site densities. The quantity $\hat{d}=\hat{n}_{1}-\hat{n}_{2}$ can be interpreted as the dipole moment of our simplified model of a diatomic system and its expectation value $d(t)=\langle\psi(t)|\hat{d}| \psi(t)\rangle$ uniquely determines the on-site densities $n_{1}(t)$ and $n_{2}(t)$ if the total number of particles is fixed. In the following, in particular for TDDFT, we will use the dipole moment $d(t)$ as the basic "density variable."

Since the dipole moment operator $\hat{d}$ is odd under reflection (interchange of site indices), it has nonzero matrix elements only between states of different parity. In particular, $\hat{d}$ connects the ground state $|g\rangle$ of Eq. (3a) only to the first excited state $\left|e_{1}\right\rangle$,

$$
d_{g e}=\left\langle g|\hat{d}| e_{1}\right\rangle=\frac{2}{\sqrt{1+\beta_{+}^{2}}},
$$

while the matrix element of $\hat{d}$ between the ground state and the second excited state vanishes, $\left\langle g|\hat{d}| e_{2}\right\rangle=0$.

Now we are ready to discuss Rabi oscillations in the Hubbard dimer. Let us consider the evolution of the systems from its ground state $|\psi(0)\rangle=|g\rangle$ under the action of a time periodic potential

$$
\Delta v(t)=2 \mathcal{E}_{0} \sin (\omega t) .
$$

The Rabi regime of dynamics occurs when the frequency $\omega$ of a sufficiently weak driving field approaches the frequency $\omega_{0}$ of 


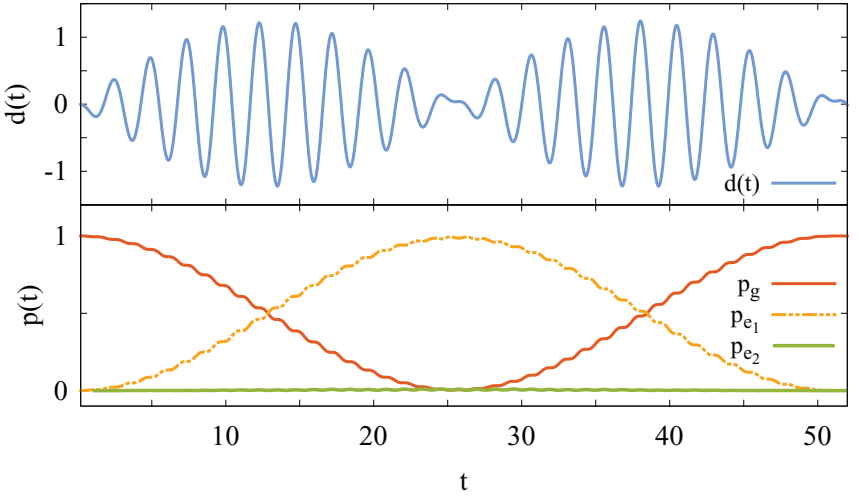

FIG. 1. (Color online) Rabi oscillations for resonant laser $\omega=$ $\omega_{0}=2.56 \mathrm{~T}$. Upper panel: dipole moment $d(t)$. Lower panel: population of ground state $p_{g}=|\langle g \mid \psi\rangle|^{2}$ (solid red), first excited state $p_{e_{1}}=\left|\left\langle e_{1} \mid \psi\right\rangle\right|^{2}$ (dotted orange), and second excited state $p_{e_{2}}=$ $\left|\left\langle e_{2} \mid \psi\right\rangle\right|^{2}$ (dashed green). Time is given in units of $1 / T$, where $T$ is the hopping parameter.

the main dipole resonance. In our case this corresponds to the frequency $\omega \sim \omega_{0}=E_{e_{1}}-E_{g}$ close to the energy difference between ground and first excited states, and the amplitude $\mathcal{E}_{0} \ll \omega_{0} / d_{g e}$.

Figure 1 shows resonant dynamics of the dipole moment and state populations obtained by the numerical propagation of Eq. (2) for a moderately strong interaction $U=T$, frequency $\omega=\omega_{0}=2.56 \mathrm{~T}, d_{g e}=1.23$, amplitude $\mathcal{E}_{0}=0.1 \mathrm{~T}$, and fixed electron number $N=n_{1}+n_{2}=2$. We see that the populations $p_{g}=|\langle g \mid \psi\rangle|^{2}$ and $p_{e_{1}}=\left|\left\langle e_{1} \mid \psi\right\rangle\right|^{2}$ of the ground and the first exited state oscillate between zero and one, while the second excited state stays practically unpopulated, $p_{e_{2}}=$ $\left|\left\langle e_{2} \mid \psi\right\rangle\right|^{2} \approx 0$. The dipole moment shows fast oscillations at the driving frequency $\omega$ superimposed with slow oscillations of the envelope at the Rabi frequency $\Omega_{R}=d_{g e} \mathcal{E}_{0}$. The maximal value of the dipole moment $\left|d_{\max }\right|=d_{g e}=1.23$ is reached at $1 / 4$ and $3 / 4$ of the Rabi cycle when the ground and the first excited states have equal populations of $1 / 2$.

The main characteristic feature of the Rabi regime is a strong variation of the state populations. It is this feature which makes the description of Rabi oscillations one of the most difficult cases for TDDFT [18,29]. In the rest of this paper we discuss the TDDFT approach to the Rabi dynamics for our simple two-site system.

\section{TIME-DEPENDENT KOHN-SHAM EQUATIONS FOR A HUBBARD DIMER}

In the present two-electron case the KS system corresponds to two noninteracting particles which reproduce the timedependent dipole moment $d(t)$ of the interacting system. The KS Hamiltonian has the form of Eq. (1) but with no interaction $(U=0 \mathrm{~T})$ and the external potential $\Delta v^{s}$ is chosen such that the correct time-dependent density of the interacting system is reproduced.

For a singlet state both KS particles occupy the same one-particle $\mathrm{KS}$ orbital, which is described by two on-site amplitudes $\varphi_{1}(t)$ and $\varphi_{2}(t)$. Therefore the time-dependent KS equations reduce to a single $2 \times 2$ one-particle Schrödinger equation of the form

$$
\begin{aligned}
i \partial_{t} \varphi_{1} & =-T \varphi_{2}+\frac{\Delta v_{s}}{2} \varphi_{1}, \\
i \partial_{t} \varphi_{2} & =-T \varphi_{1}-\frac{\Delta v_{s}}{2} \varphi_{2} .
\end{aligned}
$$

As our dynamics starts from the ground state, Eq. (9) has to be solved with the initial condition $\varphi_{1}(0)=\varphi_{2}(0)=1 / \sqrt{2}$ which corresponds to the noninteracting KS ground state. By definition the KS potential $\Delta v_{s}(t)$ entering Eq. (9) produces a prescribed (interacting) dipole moment. In the present case this KS potential can be found explicitly as a functional of the density $d(t)[10,28]$,

$$
\Delta v_{s}[d]=-\frac{\ddot{d}+4 T^{2} d}{\sqrt{4 T^{2}\left(4-d^{2}\right)-\dot{d}^{2}}} .
$$

It is important to note that the functional $\Delta v_{s}[d]$ is given by Eq. (10) only if the system evolves from, and remains sufficiently close to, the ground state. More precisely, it is shown in Ref. [10] that the functional form of Eq. (10) is valid as long as the condition $\left|\arg \left(\varphi_{1}\right)-\arg \left(\varphi_{2}\right)\right|<\pi / 2$ is satisfied during the course of the evolution. If the opposite inequality holds, the overall sign on the right-hand side of Eq. (10) has to be changed from - to + . Moreover, the sign changes every time the line $\left|\arg \left(\varphi_{1}\right)-\arg \left(\varphi_{2}\right)\right|=\pi / 2$ is crossed. In terms of the dipole moment, crossing this line corresponds to a vanishing expression under the square root in Eq. (10) [30]. The above behavior can be viewed as a manifestation of the initial state and history dependence in TDDFT [10,31].

The exact KS potential can be calculated by inserting into Eq. (10) the exact dipole moment $d(t)$ obtained from a numerical solution of the many-body Schrödinger equation (2). In order to get the Hxc potential we subtract the physical external potential $\Delta v$ from the KS potential,

$$
\Delta v_{\mathrm{Hxc}}=\Delta v_{s}-\Delta v \text {. }
$$

The time dependence of the exact $\Delta v_{\mathrm{Hxc}}$ which corresponds to the dipole moment $d(t)$ presented in Fig. 1 (i.e., to the regime of resonant Rabi oscillations, described in Sec. II) is shown in the top panel of Fig. 3.

In practice the exact Hxc functional is unknown and one has to rely on approximations. The simplest and the most common approximation in TDDFT is based on the adiabatic assumption for xc effects. Below we present and test the adiabatic approximation for our model system.

\section{A. Adiabatically exact functional}

To construct the adiabatic approximation for the Hubbard dimer we first find the exact ground-state $\mathrm{Hxc}$ functional by the Levy-Lieb constrained search, i.e., we perform an exhaustive search over the space of all allowed two-particle wave functions $\Psi$ that yield a given dipole moment $d$ to find the Hohenberg-Kohn energy functional $F_{\mathrm{HK}}[d]$,

$$
F_{\mathrm{HK}}[d]=\min _{\Psi \rightarrow d}\langle\Psi|\hat{T}+\hat{U}| \Psi\rangle,
$$


where $\hat{T}$ and $\hat{U}$ are operators of the kinetic energy and the interaction energy, i.e., the first and the second terms in the Hamiltonian (1), respectively.

The exact ground-state Hxc potential is given by the derivative of the Hxc energy with respect to the dipole moment,

$$
\Delta v_{\mathrm{Hxc}}^{g s}[d]=2 \frac{\partial}{\partial d}\left(F_{\mathrm{HK}}[d]-T^{s}[d]\right),
$$

where $T^{s}[d]$ is the kinetic energy functional that is defined by Eq. (12) with $U=0 \mathrm{~T}$. More details on this construction can be found in the Appendix.

In the adiabatically exact approximation the exact groundstate Hxc potential of Eq. (13) is used in the time-dependent KS equations, i.e., the Hxc potential at time $t$ is calculated by inserting the instantaneous value of $d(t)$ into the ground-state functional

$$
\Delta v_{\mathrm{Hxc}}^{\mathrm{ad}}[d](t)=\Delta v_{\mathrm{Hxc}}^{g s}[d(t)] .
$$

We note that the adiabatically exact Hxc potential has also been found numerically for real-space one-dimensional two-electron systems in Refs. [11,19,20] using the iterative procedure introduced in Ref. [20]. A big advantage of the present simple model is that we know explicitly the functional dependence of the exact ground-state Hxc potential (see Appendix), i.e., we do not need any a priori knowledge of the time-dependent density.

To test the performance of the adiabatically exact functional in the regime of Rabi oscillations we propagate selfconsistently the KS equations with $v_{\mathrm{Hxc}}^{\mathrm{ad}}[d](t)$ for the same parameters as in Sec. II.

The results of the propagation confirm a general conclusion of Ref. [18] about the presence of an artificial dynamical detuning in the description of Rabi oscillations using adiabatic functionals. In Fig. 2 we compare the evolution of the exact dipole moment $d(t)$ (blue) with the dipole moment $d_{\mathrm{ad}}(t)$ (red) obtained from KS equations with the adiabatically exact Hxc potential for resonant (upper panel) and slightly detuned

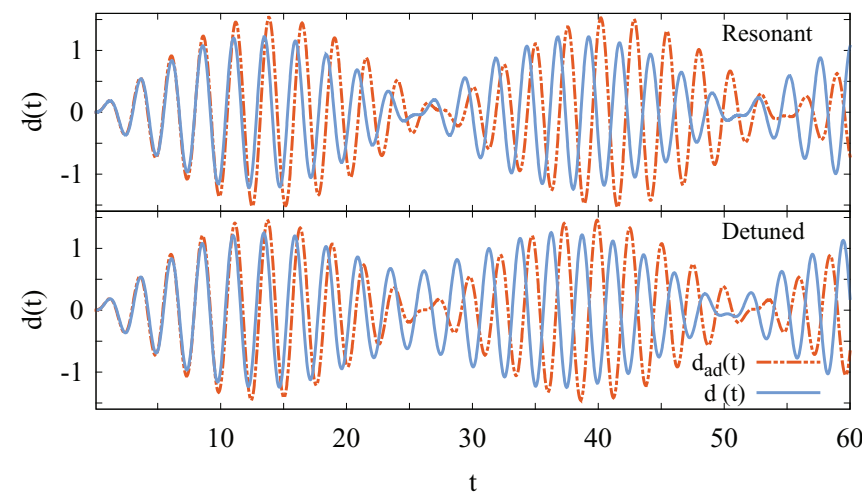

FIG. 2. (Color online) Upper panel: $d(t)$ (solid blue) in the presence of a laser of frequency $\omega=\omega_{0}=2.56 \mathrm{~T}$, compared to $d_{\mathrm{ad}}(t)$ (dotted red) propagated using the exact ground-state functional $\Delta v_{\mathrm{Hxc}}^{g s}[d]$ in the presence of a laser resonant with the adiabatically exact linear response frequency $\omega_{\text {ad }}^{L R}=2.60 \mathrm{~T}$. Lower panel: $d(t)$ for slightly detuned laser $\omega=\omega_{0}+0.03 \mathrm{~T}$ (solid blue) compared to $d_{\mathrm{ad}}(t)$ using $\omega=\omega_{\mathrm{ad}}^{L R}+0.03 \mathrm{~T}$ (dotted red). Time is given in units of the inverse of the hopping parameter $T$. (lower panel) applied lasers. The upper panel shows the dynamics at resonant conditions when the frequency $\omega$ of the driving field is equal to the frequency $\omega_{\text {res }}$ of the main dipole resonance. In the exact interacting system this frequency is obviously $\omega_{\text {res }}=\omega_{0}$, while in the approximate TDDFT it is approximation dependent, and should be determined consistently as the frequency $\omega_{\text {res }}=\omega_{L R}$ of the corresponding linear response resonance. At first sight, the function $d_{\mathrm{ad}}(t)$ resulting from TDDFT with the adiabatic Hxc potential (upper panel in red in Fig. 2) looks qualitatively similar to the exact $d(t)$ (upper panel in blue in Fig. 2). However, there is a deep difference in the underlying microscopic dynamics. The physical system returns to its initial state after two periods of the dipole moment's envelope, which corresponds to the Rabi period of $T_{\mathrm{R}}=\frac{2 \pi}{\mathcal{E}_{0} d_{g e}}=51.101 / \mathrm{T}$. In contrast, the microscopic period of the KS system with the adiabatic Hxc potential coincides with that of the dipole moment, which is the characteristic feature of detuned Rabi oscillations. In fact, the KS Rabi dynamics is always internally detuned by the presence of the adiabatic potential $\Delta v_{\mathrm{Hxc}}^{g s}[d(t)]$ which depends on the instantaneous density [18]. While this important difference is hidden in the case of resonant dynamics of the dipole moment, it is revealed immediately when the driving frequency is a bit shifted (detuned) from the exact resonance. The dipole moments $d(t)$ and $d_{\mathrm{ad}}(t)$ for a slightly detuned driving field with $\omega=\omega_{\text {res }}+0.03$ are presented in the lower panel in Fig. 2. The exact dipole moment $d(t)$ develops a "neck" at $t \sim T_{\mathrm{R}}$ showing that the actual physical period is indeed $T_{\mathrm{R}} \approx 50$. On the other hand, the function $d_{\mathrm{ad}}(t)$ is practically unaffected by the external detuning because the KS system, being already strongly detuned internally, is insensitive to small external variations of the driving frequency. This qualitative failure of the adiabatic approximation clearly demonstrates the important role of xc memory effects in the correct description of Rabi oscillations.

To further quantify nonadiabatic effects in the Rabi regime we extract a nonadiabatic contribution to the total Hxc potential. Namely, we subtract the adiabatic potential $\Delta v_{\mathrm{Hxc}}^{g s}[d(t)]$ evaluated at the exact dipole moment $d(t)$ from the exact $\Delta v_{\mathrm{Hxc}}(t)$ defined by Eqs. (11) and (10). In Fig. 3 we present

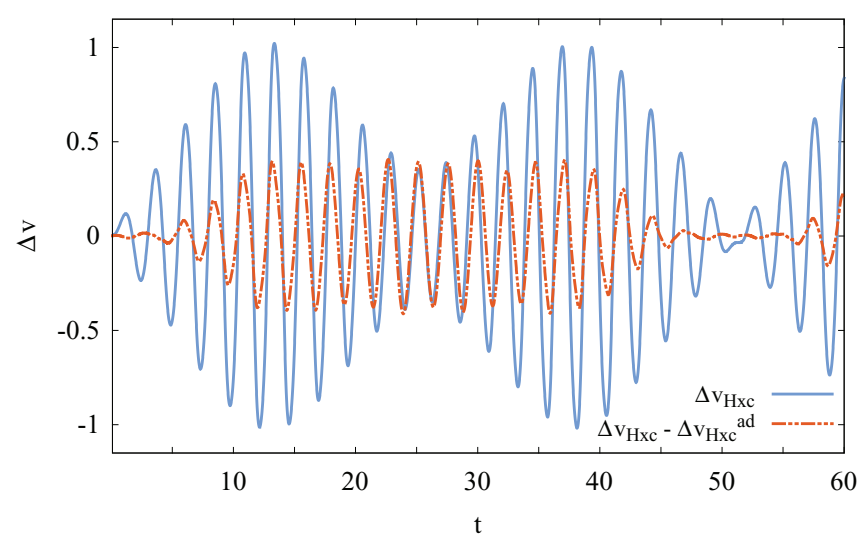

FIG. 3. (Color online) Time-dependent Hxc potential $\Delta v_{\mathrm{Hxc}}(t)$ (in units of the hopping parameter $T$ ) (solid blue) and its nonadiabatic contribution defined as $\Delta v_{\mathrm{Hxc}}(t)-\Delta v_{\mathrm{Hxc}}^{g s}[d(t)]$ (in units of the hopping parameter $T$ ) (dotted red). Time is given in units of $1 / T$. 
the nonadiabatic part of Hxc potential together with the exact $\Delta v_{\mathrm{Hxc}}(t)$. The nonadiabatic contribution to $\Delta v_{\mathrm{Hxc}}(t)$ turns out to be more than double the amplitude of the external potential and in fact as large as the Hxc potential itself during a significant part of the Rabi cycle. Not surprisingly, the nonadiabatic effects are small at the beginning and at the end of the Rabi cycle when the ground-state population is dominant and the system is close to the linear response regime. But they grow fast when the system is driven away from the ground state and they remain large for a large part of the Rabi cycle. It is interesting to notice that, centered around $T_{R} / 2$, there is a long period of time during which the amplitude of the adiabatic effects remains almost constant (see Fig. 3).

Apparently a better approximation for the xc potential is needed to capture nonadiabatic effects relevant to describe Rabi oscillations. In the next section we propose an explicit nonadiabatic density functional based on a two-level description of the interacting system.

\section{TIME-DEPENDENT xc POTENTIAL IN THE TWO-LEVEL APPROXIMATION}

In general, the Hxc functional $\Delta v_{\mathrm{Hxc}}[d]$ can be found via Eq. (11) if we know the external potential as a functional of $d(t)$. In general the problem of finding the functional $\Delta v[d]$ is highly nontrivial. In our simple model it is the presence of interactions what makes finding $\Delta v[d]$ demanding. Fortunately in some cases like the Rabi oscillations the problem is simplified dramatically because the behavior of the system is close to that of an effective two-level system.

Let us assume that the second excited state $\left|e_{2}\right\rangle$ is not participating in the dynamics. We write the many-body Schrödinger equation (2) in the two-level approximation as

$$
\begin{aligned}
& i \partial_{t} \psi_{g}(t)=E_{g} \psi_{g}(t)+d_{g e} \frac{\Delta v(t)}{2} \psi_{e}(t), \\
& i \partial_{t} \psi_{e}(t)=E_{e_{1}} \psi_{e}(t)+d_{g e} \frac{\Delta v(t)}{2} \psi_{g}(t),
\end{aligned}
$$

where $\psi_{g}(t)=\langle g \mid \psi(t)\rangle$ and $\psi_{e}(t)=\left\langle e_{1} \mid \psi(t)\right\rangle$ are the projections of the time-dependent wave function onto the ground and first excited state, respectively.

By rotating the basis we can represent Eq. (15) in the form of a Schrödinger equation for one particle on an effective "two-site lattice." In other words, Eq. (15) is unitarily equivalent to Eq. (9) with hopping constant $\omega_{0} / 2$ and external potential given by $d_{g e} \Delta v / 2$. Using this mapping and the $\mathrm{KS}$ potential $\Delta v_{s}$ of Eq. (10) we can immediately write the external potential $\Delta v$ of the interacting system as a functional of the dipole moment $d$,

$$
\Delta v^{2 L}[d]=\frac{(-1)^{p}}{d_{g e}}\left(\frac{\ddot{d}+\omega_{0}^{2} d}{\sqrt{\omega_{0}^{2}\left(d_{g e}^{2}-d^{2}\right)-\dot{d}^{2}}}\right),
$$

where the integer $p$ counts how many times the square root turns into zero during the evolution. The factor $(-1)^{p}$ accounts for the sign changes explained after Eq. (10).

In order to find the Hxc potential $\Delta v_{\mathrm{Hxc}}[d]$ as a functional of the dipole moment we substitute the external potential $\Delta v$ of Eq. (16) and the KS potential of Eq. (10) into Eq. (11):

$$
\begin{aligned}
\Delta v_{\mathrm{Hxc}}^{2 L}[d]= & -\frac{\ddot{d}+4 T^{2} d}{\sqrt{4 T^{2}\left(4-d^{2}\right)-\dot{d}^{2}}} \\
& -\frac{(-1)^{p}}{d_{g e}}\left(\frac{\ddot{d}+\omega_{0}^{2} d}{\sqrt{\omega_{0}^{2}\left(d_{g e}^{2}-d^{2}\right)-\dot{d}^{2}}}\right) .
\end{aligned}
$$

This expression is one of the main results of the present paper. It provides us with an explicit fully nonadiabatic density functional which, by construction, should correctly describe the Rabi oscillations. It is worth emphasizing that the functional Eq. (17) contains history dependence via the integer $p$ in the second term.

One can easily check that the nonlinear functional $\Delta v_{\mathrm{Hxc}}^{2 L}$ of Eq. (17) produces the exact dynamic xc kernel in the linear response regime. The formally exact $\mathrm{Hxc}$ functional in the linear response can be written as follows:

$$
\Delta v_{\mathrm{Hxc}}^{L R}[d](\omega)=\left[\chi_{s}^{-1}(\omega)-\chi^{-1}(\omega)\right] d=f_{\mathrm{Hxc}}(\omega) d,
$$

where $\chi_{s}(\omega)$ and $\chi(\omega)$ are the density response functions for the KS and the interacting system, respectively. By definition the term in parentheses is the exact exchange-correlation kernel $f_{\mathrm{Hxc}}(\omega)$. Since the eigenfunctions for the Hubbard dimer are known, Eqs. (3) and (5), we can write the exact response functions $\chi(\omega)$ in the Lehmann representation [32], and substitute it into Eq. (18). The result takes the following form:

$$
\Delta v_{\mathrm{Hxc}}^{L R}[d](\omega)=\left(T-\frac{\omega_{0}}{2 d_{g e}^{2}}+\left[\frac{1}{4 T}-\frac{1}{2 \omega_{0} d_{g e}^{2}}\right] \omega^{2}\right) d .
$$

It is now straightforward to see that this equation is identical to the linearized version of the approximate functional $\Delta v_{\mathrm{Hxc}}^{2 L}[d]$ defined by Eq. (17) with $p=0$. In other words our approximation becomes exact in the linear regime. This nice property is not accidental because the functional of Eq. (17) is based on the two-level approximation. In the linear response regime, the symmetric Hubbard dimer becomes an effective two-level system because the dipole transition matrix element between the ground state $|g\rangle$ and the second excited state $\left|e_{2}\right\rangle$ vanishes.

A subtle property of the nonadiabatic functional Eq. (17) is the dependence on the second time derivative $\ddot{d}$ of the dipole moment. The presence of $\ddot{d}$ does not mean that the xc potential assumes a dependence on the future. In general the existence theorem for TDDFT on a lattice [10] requires the second time derivative of the density to be continuous. Therefore $\ddot{d}$ can be calculated as a left limit for any time greater than the initial time, $t>0$. At $t=0$ the value of $\ddot{d}(0)$ is determined by the initial value of the external potential as follows:

$$
\ddot{d}(0)=-d_{d e} \Delta v(0) \sqrt{\omega_{0}^{2}\left[d_{g e}^{2}-d^{2}(0)\right]-\dot{d}^{2}(0)}-\omega_{0}^{2} d(0),
$$

where the $d(0)$ and $\dot{d}(0)$ are fixed by the KS initial state

$$
\begin{aligned}
& d(0)=2\left[\left|\varphi_{1}(0)\right|^{2}-\left|\varphi_{2}(0)\right|^{2}\right], \\
& \dot{d}(0)=-4 T \operatorname{Im}\left[\varphi_{1}^{*}(0) \varphi_{2}(0)\right] .
\end{aligned}
$$




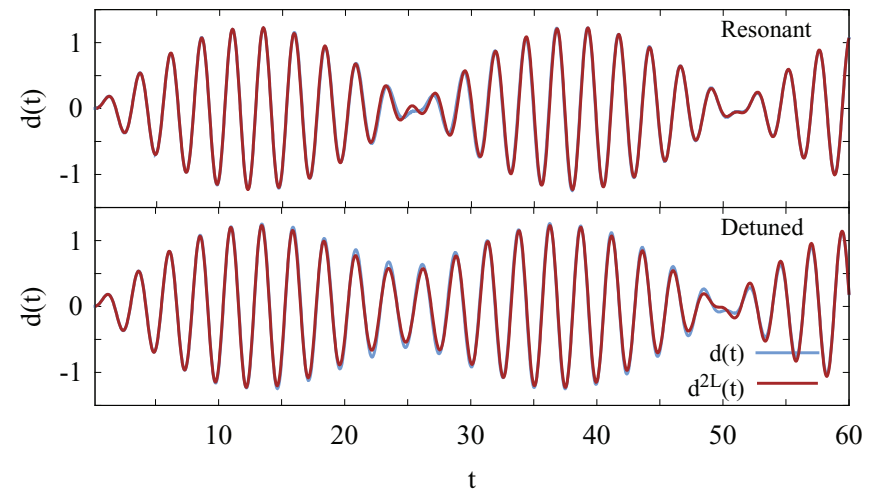

FIG. 4. (Color online) Upper panel: $d(t)$ (solid blue) for resonant laser frequency $\omega=\omega_{0}=2.56 \mathrm{~T}$ compared to two-level approximation $d^{2 L}(t)$ (dashed brown) using $\Delta v_{\mathrm{Hxc}}^{2 L}$, Eq. (17), and same laser frequency. Lower panel: $d(t)$ (solid blue) and $d^{2 L}(t)$ (dashed red) for slightly detuned laser $\omega=\omega_{0}+0.03 \mathrm{~T}$ (same detuning as lower panel in Fig. 2). Time is given in units of $1 / T$.

After these preliminaries we can plug the Hxc potential Eq. (17) into the KS equations and propagate them selfconsistently to test the performance of our nonadiabatic approximation. It is, however, clear that the functional $\Delta v_{\mathrm{Hxc}}^{2 L}[d]$, by construction, should exactly reproduce the results of the two-level approximation to the full interacting problem. Therefore TDDFT with the Hxc potential of Eq. (17) is as accurate as the two-level approximation itself.

In Fig. 4 we compare the exact resonant Rabi dynamics of the dipole moment with that obtained in the two-level approximation or, alternatively, by solving self-consistently the KS equations with the potential $\Delta v_{\mathrm{Hxc}}^{2 L}[d]$. The exact and approximate dipole moments are practically on top of each other. The nonadiabatic functional of Eq. (17) excellently reproduces Rabi oscillations for a resonant excitation. Apparently it also works perfectly for detuned Rabi dynamics provided the detuning is not too large. Another nice property of this approximation is that it becomes essentially exact for a sufficiently weak nonresonant driving potential which corresponds to the linear response regime.

\section{CONCLUSION}

We use a Hubbard dimer to analyze, both qualitatively and quantitatively, the nonadiabatic features present in the TDDFT functional. For this model system the exact Kohn-Sham potential is analytic and moreover, the exact ground-state functional can be found by Levy-Lieb constrained search. The latter is propagated self-consistently to study the performance of the adiabatic approximation. We show that nonadiabaticity is crucial to properly capture the physics of resonant and nearly detuned Rabi oscillations. The observed nonadiabatic features grow as the population of the excited state is rising to its maximum, becoming even larger than the external potential. Lack of these features in adiabatic functionals causes them to fail to describe Rabi dynamics, missing both frequency and amplitude of the physical dipole moment. Taking advantage of the fact that under the action of a resonant laser the system behaves as an effective two-level one, we derive an explicit nonadiabatic functional that accurately reproduces resonant and slightly detuned Rabi oscillations. This fully nonadiabatic functional incorporates explicitly the initial-state dependence and becomes exact in the linear response regime.

The present work was focused on the TDDFT description of Rabi oscillations in a minimal model system. While the construction of the quasiexact TDDFT for this minimalistic system was nontrivial, the nonadiabatic part of the Hxc potential has been found to have a relatively simple structure (see Fig. 3): While nonadiabaticity is small close to the beginning and the end of the Rabi cycle, its amplitude is significant but almost constant throughout a large middle part of the Rabi cycle. This might be a useful observation when aiming to construct nonadiabatic TDDFT functionals applicable to realistic systems.

\section{ACKNOWLEDGMENTS}

We acknowledge financial support from the European Research Council Advanced Grant DYNamo (Grant No. ERC-2010-AdG-267374), Spanish Grant (Grant No. FIS2010-21282-C02-01), Grupos Consolidados UPV/EHU del Gobierno Vasco (Grant No. IT578-13), and Ikerbasque and the European Commission projects CRONOS (Grant No. 280879-2 CRONOS CP-FP7).

\section{APPENDIX: EXACT HOHENBERG-KOHN FUNCTIONAL OF THE TWO-SITE HUBBARD MODEL BY CONSTRAINED SEARCH}

In this Appendix we briefly describe how one can construct the exact Hohenberg-Kohn functional (12) for two electrons in the two-site Hubbard model by carrying out the constrained search as suggested by Levy [25,26] and Lieb [27].

The Hilbert space for two fermions on two sites is of dimension six and separates into a singlet and a triplet sector of dimension three each. Since for any value of $d$, the ground state of $\hat{H}_{0}=\hat{T}+\hat{U}$ is a singlet, we may restrict the search in Eq. (12) to singlet wave functions only. As a basis for the singlet sector we use the eigenstates of Eq. (3). Then the most general singlet state may be written as

$$
|\Psi\rangle=A_{1}|g\rangle+A_{2}\left|e_{1}\right\rangle+A_{3}\left|e_{2}\right\rangle,
$$

where we can, without loss of generality, choose the coefficients $A_{i}$ to be real. In the chosen basis, the expectation value of $\hat{H}_{0}=\hat{T}+\hat{U}$ takes the simple form

$$
\left\langle\Psi\left|\hat{H}_{0}\right| \Psi\right\rangle=\langle\Psi|\hat{T}+\hat{U}| \Psi\rangle=E_{g} A_{1}^{2}+E_{e_{1}} A_{2}^{2}+E_{e_{2}} A_{3}^{2},
$$

where the eigenvalues $E_{g}, E_{e_{1}}$, and $E_{e_{2}}$ of the basis functions are given by Eq. (5).

The expansion coefficients $A_{i}$ in Eq. (A1) are not independent. The normalization condition of the wave function $|\Psi\rangle$ leads to

$$
A_{1}^{2}+A_{2}^{2}+A_{3}^{2}=1 .
$$

In the constrained search we also have to make sure that we are only searching over wave functions which yield a given "density" $d$. This gives a second condition on the coefficients 


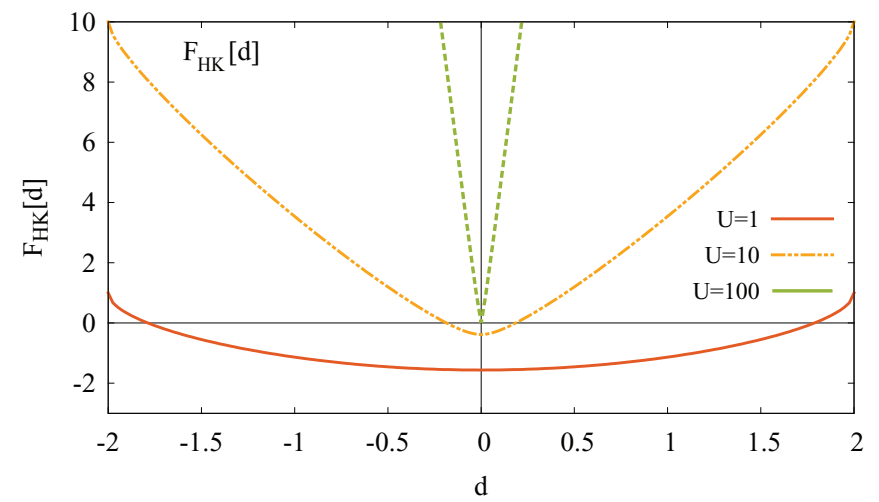

FIG. 5. (Color online) Exact Hohenberg-Kohn density functional $F_{\mathrm{HK}}[d]$ (in units of the hopping parameter $T$ ) for different Hubbard strengths $U$.

which reads

$$
d=\left\langle\Psi\left|\hat{n_{1}}-\hat{n}_{2}\right| \Psi\right\rangle=4 \frac{A_{1}+\beta_{+} A_{3}}{\sqrt{1+\beta_{+}^{2}}} A_{2},
$$

where $\beta_{+}$is given by Eq. (4). We can use Eqs. (A3) and (A4) to eliminate two of the coefficients, say $A_{2}$ and $A_{3}$, in the constrained search which then becomes a minimization in a single variable, i.e.,

$$
F_{\mathrm{HK}}(d)=\min _{A_{1}}\left\langle\Psi\left(A_{1}, d\right)|\hat{T}+\hat{U}| \Psi\left(A_{1}, d\right)\right\rangle
$$

In general, this minimization has to be carried out numerically. In Fig. 5 we show $F^{\mathrm{HK}}$ as a function of the dipole moment for various values of $U$. We note that $F^{\mathrm{HK}}(d)$ is always minimal at $d=0$. For large values of $U$ the slope of $F^{\mathrm{HK}}(d)$ changes rapidly as one crosses from negative to positive values of $d$. For vanishing interaction $U=0$, the minimization can be carried out fully analytically. The resulting functional, the noninteracting kinetic energy, reads

$$
T_{s}(d)=\min _{\Psi \rightarrow d}\langle\Psi|\hat{T}| \Psi\rangle=2 T\left(1-2 \sqrt{1-\frac{d^{2}}{8}}\right) .
$$

The Hartree-exchange-correlation energy then is given by

$$
E_{\mathrm{Hxc}}(d)=F_{\mathrm{HK}}(d)-T_{s}(d)
$$

and the corresponding Hxc potential can be easily obtained by differentiation.
[1] M. Marques, N. T. Maitra, F. Nogueira, E. Gross, and A. Rubio, Fundamentals of Time-Dependent Density Functional Theory, Lecture Notes in Physics Vol. 837 (Springer, Heidelberg, 2012).

[2] M. E. Casida, J. Mol. Struct.: THEOCHEM 914, 3 (2009).

[3] E. Livshits and R. Baer, Phys. Chem. Chem. Phys. 9, 2932 (2007).

[4] Neepa T. Maitra, J. Chem. Phys. 122, 234104 (2005).

[5] O. Gritsenko and E. J. Baerends, J. Chem. Phys. 121, 655 (2004).

[6] D. J. Tozer, J. Chem. Phys. 119, 12697 (2003).

[7] N. T. Maitra, F. Zhang, R. J. Cave, and K. Burke, J. Chem. Phys. 120, 5932 (2004).

[8] N. Helbig, J. Fuks, I. Tokatly, H. Appel, E. Gross, and A. Rubio, Chem. Phys. 391, 1 (2011).

[9] P. Elliott, S. Goldson, C. Canahui, and N. T. Maitra, Chem. Phys. 391, 110 (2011).

[10] M. Farzanehpour and I. V. Tokatly, Phys. Rev. B 86, 125130 (2012).

[11] P. Elliott, J. I. Fuks, A. Rubio, and N. T. Maitra, Phys. Rev. Lett. 109, 266404 (2012).

[12] N. T. Maitra, K. Burke, and C. Woodward, Phys. Rev. Lett. 89, 023002 (2002).

[13] P. Elliott and N. T. Maitra, Phys. Rev. A 85, 052510 (2012).

[14] M. Ruggenthaler, S. E. B. Nielsen, and R. van Leeuwen, Phys. Rev. A 88, 022512 (2013).

[15] C. Ullrich, Time-Dependent Density-Functional Theory (Oxford University Press, Oxford, 2012).

[16] M. Marques and A. Rubio, Phys. Chem. Chem. Phys. 11, 4436 (2009).

[17] Chem. Phys. 391 (1) (2011), special issue, guest-edited by R. Baer, L. Kronik, and S. Kümmel.
[18] J. I. Fuks, N. Helbig, I. V. Tokatly, and A. Rubio, Phys. Rev. B 84, 075107 (2011).

[19] J. I. Fuks, P. Elliott, A. Rubio, and N. T. Maitra, J. Phys. Chem. Lett. 4, 735 (2013).

[20] M. Thiele, E. K. U. Gross, and S. Kümmel, Phys. Rev. Lett. 100, 153004 (2008).

[21] J. D. Ramsden and R. W. Godby, Phys. Rev. Lett. 109, 036402 (2012).

[22] D. J. Carrascal and J. Ferrer, Phys. Rev. B 85, 045110 (2012).

[23] C. A. Ullrich, J. Chem. Phys. 125, 234108 (2006).

[24] C. Verdozzi, Phys. Rev. Lett. 101, 166401 (2008).

[25] M. Levy, Proc. Natl. Acad. Sci. USA 76, 6062 (1979).

[26] M. Levy, Phys. Rev. A 26, 1200 (1982).

[27] E. Lieb, Int. J. Quantum Chem. 24, 243 (1983).

[28] Yonghui Li and C. A. Ullrich, J. Chem. Phys. 129, 044105 (2008).

[29] M. Ruggenthaler and D. Bauer, Phys. Rev. Lett. 102, 233001 (2009).

[30] Since the whole right-hand side in this equation is proportional to the time derivative of the denominator, turning the square root into zero does not lead to a divergence because the numerator vanishes at the same time. Also, for any physical evolution the expression under the square root always stays non-negative as $|\dot{d}|$ for a lattice is properly bounded from above $[10,24,33]$.

[31] N. T. Maitra, in Time-Dependent Density Functional Theory, Lecture Notes in Physics Vol. 706 (Springer, Heidelberg, 2011).

[32] G. F. Giuliani and G. Vignale, Quantum Theory of the Electron Liquid (Cambridge University Press, Cambridge, 2005).

[33] I. V. Tokatly, Phys. Rev. B 83, 035127 (2011). 